INPLASY

PROTOCOL

To cite: Gao et al. TCM nonpharmacological treatment of premenstrual dysphoric disorder: A protocol for systematic review and metaanalysis. Inplasy protocol 202140058. doi: 10.37766/inplasy2021.4.0058

Received: 09 April 2021

Published: 10 April 2021

Corresponding author: Mingzhou Gao

gmingzhou@163.com

Author Affiliation: Shandong University of traditional Chinese Medicine

Support: NCFS.

Review Stage at time of this submission: Preliminary searches.

Conflicts of interest:

None declared.

\section{TCM nonpharmacological treatment of premenstrual dysphoric disorder: A protocol for systematic review and meta-analysis}

Gao, M1; Sun, H2; An, L3; Zhang, X4; Wei, F5; Zhao, Z6; Cai, Y7; Qiao, M8; Gao, D9\%.

Review question / Objective: The aim of this systematic review and meta-analysis of trials is to evaluate the effectiveness and safety of TCM nonpharmacological interventions for PMDD.

Condition being studied: Premenstrual Dysphoric Disorder (PMDD) is becoming more and more common among women of childbearing age. Traditional Chinese medicine (TCM) nonpharmacological interventions are gaining increasing popularity for PMDD treatment, yet their comparative effectiveness and safety remains unclear. Therefore, this study will aim to compare their effectiveness and safety for PMDD.

INPLASY registration number: This protocol was registered with the International Platform of Registered Systematic Review and Meta-Analysis Protocols (INPLASY) on 10 April 2021 and was last updated on 10 April 2021 (registration number INPLASY202140058).

\section{INTRODUCTION}

Review question / Objective: The aim of this systematic review and meta-analysis of trials is to evaluate the effectiveness and safety of TCM nonpharmacological interventions for PMDD.
Condition being studied: Premenstrual Dysphoric Disorder (PMDD) is becoming more and more common among women of childbearing age. Traditional Chinese medicine (TCM) nonpharmacological interventions are gaining increasing popularity for PMDD treatment, yet their comparative effectiveness and safety remains unclear. Therefore, this study will 
aim to compare their effectiveness and safety for PMDD.

\section{METHODS}

Search strategy: The strategy will be created according to the Cochrane handbook guidelines. The established search strategy for PubMed was displayed, as follows: Search strategy in PubMed Number Entry terms : (Premenstrual dysphoric disorder OR Premenstrual syndrome OR Premenstrual disorders OR Premenstrual tension OR late luteal phase dysphoric disorder OR premenstrual tension syndrome )AND (Acupuncture OR moxibustion OR cupping OR massage OR scraping OR Taichi OR Baduanjin OR Wuqinxi).

Participant or population: Women of reproductive age diagnosed with premenstrual dysphoric disorder or premenstrual syndrome using any diagnostic method, such as DSM (III, IV, IV$R, V), I C D$ and other diagnostic tools.

Intervention: TCM non-pharmacological interventions for PMDD will be included, including acupuncture, moxibustion, cupping, massage, scraping and traditional Chinese exercise like Taichi, Baduanjin, Wuqinxi referring to related studies17. Eligible treatment can be single-drug therapy or combination therapy, and should be given for at least 2 consecutive months. Studies of pharmacological-related treatment will be excluded.

Comparator: Comparators will include placebo, and other positive interventions. Compared with the same kind of traditional Chinese medicine non pharmacological intervention measures, but in different research stages, the selection of acupoints was taken as the same node in network analysis.

Study designs to be included: The randomized controlled trials (RCTs) will be only included and no language restrictions. Studies with non-RCT design, quasiexperiment design, missing data, duplicate publications, animal experiments and reviews or case reports will be excluded.

Eligibility criteria: The PICOS (participant, intervention, comparison, and study design) principle has been applied in the study design.

Information sources: The following electronic databases will be searched: Web of Science, PubMed, Embase, Cochrane Library, China National Knowledge Infrastructure (CNKI), China Biology Medicine disc (CBM), Wanfang Database, and Chinese Scientific Journal Database (VIP).

Main outcome(s): The change of premenstrual symptoms was the primary outcome. Due to multiple outcome measures in trials, the outcomes assessed with validated instruments will be preferred, i.e. Premenstrual Syndrome Diary (PMSD)18, PMTS, Dialy Symptom Report (DSR), COPE, Daily Record of Severity of Problems (DRSP), and visual analog scales (VAS) to assess overall PMDD symptoms, and psychiatric subscales to assess specific depressive and anxiety PMDD symptoms for inclusion in our meta-analyses, such as Beck Depression Inventory (BDI), Hamilton Depression Rating (HDRS) and Mood Disorders Questionnaire (MDQ). Additionally, we assessed harm outcomes (such as discontinuation rates and adverse events) and patient satisfaction as reported by the authors.

\section{Data management: Endnote X9.}

Quality assessment / Risk of bias analysis: Two reviewers will independently assess the risk of bias using the approach recommended by Cochrane Handbook for Systematic Reviews of Interventions21. The following risk of bias domains will be assessed: sequence generation (selection bias); allocation concealment (selection bias); blinding of participants and personnel (performance bias); blinding of outcome assessment (detection bias); incomplete outcome data (attrition bias); selective outcome reporting (reporting 
bias) and other bias. If all domains are at low risk of bias, the overall risk of bias of individual studies will be categorised as low risk of bias. Otherwise, overall risk of bias will be categorised as high risk of bias. The 'risk of bias' summary will be presented graphically.

Strategy of data synthesis: Review Manager 5.3 will be employed for metaanalysis. When statistical heterogeneity is low among the results, the fixed-effects model will be used for the meta-analysis. However, if there is considerable heterogeneity, then the random-effects model will be used to analyze the pooled effect estimates.

Subgroup analysis: If there is significant heterogeneity in the included trials, then we will conduct a subgroup analysis based on the different treatments, for example, types of acupuncture (acupuncture, manual acupuncture, electroacupuncture and so on) and different outcomes.

Sensitivity analysis: Statistical heterogeneity will be detected by the I 2 statistic and $\mathrm{X}^{2}$ test. $\mathrm{P} 50 \%$ indicates the possibility of statistical heterogeneity among the studies. If the included studies have existing heterogeneity, a randomeffects model will be used. Otherwise, we will use a fixed-effect model for calculation.

Language: None.

Country(ies) involved: China.

Keywords: PMDD; meta-analysis; cognitive impairment; affective disorder, protocol.

Contributions of each author:

Author 1 - Mingzhou Gao - The author drafted the manuscript and made contributions to the conception.

Email: gmingzhou@163.com

Author 2 - Hui Sun - The author provided searches and statistical expertise.

Author 3 - Li An - The author contributed to the development of the selection criteria.

Email: anli2000@163.com
Author 4 - Hao Zhang - The author contributed to the risk of bias assessment strategy.

Email: zhanghaojune@126.com

Author 5 - Fengqin Wei - The author read, provided feedback for the manuscript.

Email: jnwfq@126.com

Author 6 - Zhengtai Zhao - The author read, provided feedback for the manuscript.

Email: 1509935480@qq.com

Author 7 - Yawei Cai - The author read, provided feedback for the manuscript.

Email: 841765917@qq.com

Author 8 - Mingqi Qiao - The author read, provided feedback for the manuscript, and provided the funding.

Email: qmingqi@163.com

Author 9 - Dongmei Gao - The author read, provided feedback for the manuscript, and provided the funding.

Email: gcy_112@163.com 